\title{
Establishment of a Ciliogenesis- Associated Signaling Model for Polycystic Kidney Disease
}

\author{
Ling Lu ${ }^{\text {a }}$ Qiuling Liu ${ }^{b}$ Lei Zhic Xuchun Che ${ }^{c}$ Bo Xiaoc Mingxuan Cui ${ }^{d}$ \\ Mingyu $\mathrm{Yu}^{\mathrm{c}}$ Bing Yang ${ }^{\mathrm{e}}$ Jingjing Zhang ${ }^{\mathrm{b}}$ Bo Zhang ${ }^{\mathrm{c}}$
}

${ }^{a}$ Department of Nephrology, Tianjin First Central Hospital, Tianjin, China; ${ }^{b}$ Affiliated Hospital of Guangdong Medical University \& Key Laboratory of Zebrafish Model for Development and Disease of Guangdong Medical University, Zhanjiang, China; 'Department of Immunology, Tianjin Key Laboratory of Cellular and Molecular Immunology, School of Basic Medical Sciences, Tianjin Medical University, Tianjin, China; dSchool of Pharmacy, Tianjin Medical

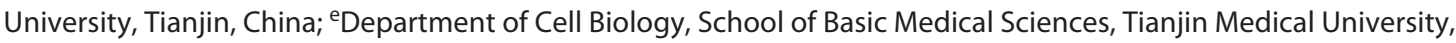
Tianjin, China

\section{Keywords}

Polycystic kidney disease · Phosphatidylinositol 4 kinase III- $\beta \cdot$ GNAS · Primary cilia

\begin{abstract}
Background: Polycystic kidney disease (PKD) represents the most prevalent inherited progressive kidney disorder in humans. Due to complexity of the genetic network behind the disease, the molecular mechanisms of PKD are still poorly understood yet. Objectives: This study aimed to develop a ciliogenesis-associated gene network for PKD patients and comprehensively understand the molecular mechanisms underlying the disease. Method: The potential hub genes were selected based on the differential expression analysis from the GEO database. Meanwhile, the primary hub genes were further elucidated by both in vivo and in vitro experiments. Results: In this study, we established a comprehensive differentially expressed genes profile (including GNAS, PI4KB, UMOD, SLC7A13, and MIOX) for PKD patients compared with the control specimen. At the same time, enrichment analysis was utilized to demonstrate that the G-pro-
\end{abstract}

karger@karger.com www.karger.com/kbr

Karger $\stackrel{\text { ' }}{5}$
(C) 2021 The Author(s)

Published by S. Karger AG, Basel

This is an Open Access article licensed under the Creative Common Attribution-NonCommercial-4.0 International License (CC BY-NC) (http://www.karger.com/Services/OpenAccessLicense), applicable to the online version of the article only. Usage and distribution for commercial purposes requires written permission. tein-related signaling and cilia assembling signaling pathways were closely associated with PKD development. The further investigations of the interaction between 2 genes (GNAS and PIAKB) with in vivo and in vitro analyses revealed that PI4KB functioned as a downstream factor for GNAS and spontaneously activated the phosphorylation of Akt into $p$ Akt for ciliogenesis in PKD formation. The PI4KB depletion mutant zebrafish model displayed a PKD phenotype as well as absence of primary cilia in the kidney. Conclusions: Collectively, our work discovered an innovative potential signaling pathway model for PKD formation, which provided a valuable insight for future study of the mechanism of this disease.

(C) 2021 The Author(s).

Published by S. Karger AG, Basel

\section{Introduction}

Polycystic kidney disease (PKD) is a group of monogenic disorders, which is characterized by relentless development and enlargement of focal kidney cysts ultimately resulting in end-stage renal disease [1]. The $2 \mathrm{ma-}$ 
jor forms of PKD are autosomal dominant PKD (ADPKD) and autosomal recessive PKD (ARPKD), in which ADPKD is more common while ARPKD is rarer and presents perinatally or in early childhood [2]. The incidence of ADPKD is approximately 1:400-1,000, which is suggested as the most common monogenic disorder [3]. ADPKD is genetically heterogeneous and associated with 2 genes identified, PKD1 (16p13.3) and PKD2 (4q21), which encode polycystin 1 (PC1) and polycystin 2 (PC2), respectively [4]. Until now, therapy for ADPKD has been directed toward limiting its complications, and the treatments are far from satisfaction.

In the past decades, the better understanding of associated signaling pathways for ADPKD has initiated various possible therapies to inhibit the formation as well as the development of kidney cysts. A reduced "dosage" of PKD proteins is thought to interfere with renal cell homeostasis and disturb multiple signaling pathways, such as G-protein-related signaling pathway, $\mathrm{Ca}^{2+}$-related pathway, mechanistic target of rapamycin, vascular endothelial growth factor, and WNT signaling [5-7]. Among these, cilia-related pathways have attracted more and more attention based on the fact that PC1 and PC2 form a heterodimeric complex in the primary cilia of renal epithelial cells through the interaction of the coiledcoil motifs in the carboxy-terminal tails of each protein [8]. More and more compelling studies exist to suggest that primary cilia localization and cilia-related signaling pathways play a central pathogenic role in PKD progress $[9,10]$.

Primary cilia are revolutionary conserved immotile organelles known for their diverse roles in organ development and cell signaling. In vertebrates, cilia exist in almost every cell type and have a variety of biological functions. The dysfunction of primary cilia could induce a wide range of developmental diseases, which are collectively referred as ciliopathies [11-13]. Phosphatidylinositol 4 kinase III- $\beta$ (PI4KB) in mammalian cells is characterized as a key regulator for the synthesis of phosphatidylinositol 4-phosphate (PI4P), which is located in the Golgi and trans-Golgi network pool [14]. Recently, it has been suggested that PI4KB is crucial for cilia-related signaling pathway, contributing to the ciliopathies [15]. However, the clear roles of PI4KB in ciliogenesis especially the cilia-related PKD formation are still controversial.

Since the molecular mechanism underlying PKD is not clear yet, the disease has brought great difficulty to clinical treatment. In this study, we established a differentially expressed gene profile for PKD patients com- pared with the control specimen. Several genes including $P I 4 K B$ demonstrated a specific expression pattern. With further investigation, $P I 4 K B$ was shown to be associated with cilia-related pathway and G-protein signaling as well as AKT signaling for kidney cyst formation. Using zebrafish as an animal model, we found both PKD phenotype and absence of primary cilia in the PI $4 K B \mathrm{mu}$ tants. All of these promising outcomes enriched the precise functions of PI4KB for PKD from a ciliogenesis aspect, which provided a tremendous help for future PKD study.

\section{Material and Methods}

\section{Data Source}

The data analyzed in this study were downloaded from the GEO database, numbered GSE7869, which contained cystic kidney tissues from 18 cases of PKD patients and 3 noncystic kidney tissues. The samples were tested on the Affymetrix Human Genome U133 Plus 2.0 Array chip platform.

\section{Differential Gene Analysis}

The raw data were normalized using the robust multi-array average method, developed by the $\log 2$ logarithm to generate the value, which was the normalized data for differential expression analysis. The differentially expressed gene analysis was based on the limma function package of the R language (version 3.5.2) [16], using the absolute logarithmic conversion of the differential expression multiple (Log2FC) value $>2$ and FDR $\leq 0.05$ as criteria for screening differentially expressed genes.

Functional Enrichment Analysis

For the obtained differentially expressed genes, we used the "clusterProfiler" function package in R language for enrichment analysis of GO (including biological process, molecular function, and cellular component) and KEGG pathways. When $p$ value is $<0.05$, we considered the corresponding entries to be significantly enriched [17].

\section{Protein-Protein Interaction Networks and Identification of}

Hub Gens

The STRING database is the one for analyzing and predicting protein functional associations and protein interactions. In this study, we utilized STRING (https://STRING-db.org/, version 11.0) to analyze protein functional associations and protein interactions [18]. The Cytoscape (version 3.7.2) was used to visualize the PPI network, and the cytoHubba plug-in in Cytoscape was performed to screen the key genes (hub genes) in the PPI network based on the algorithm of maximum neighborhood component (MNC) [19].

\section{Generation of PI4KB Mutants}

The zebrafish PI4KB loss-of-function mutants were obtained by the CRISPR-associated protein 9 (CRISPR/Cas9) system genome-editing strategy [20]. The primers designed for guide RNA (gRNA) targeting the pi4kb exon 2 were 5 '-TAATACGACTCAC- 


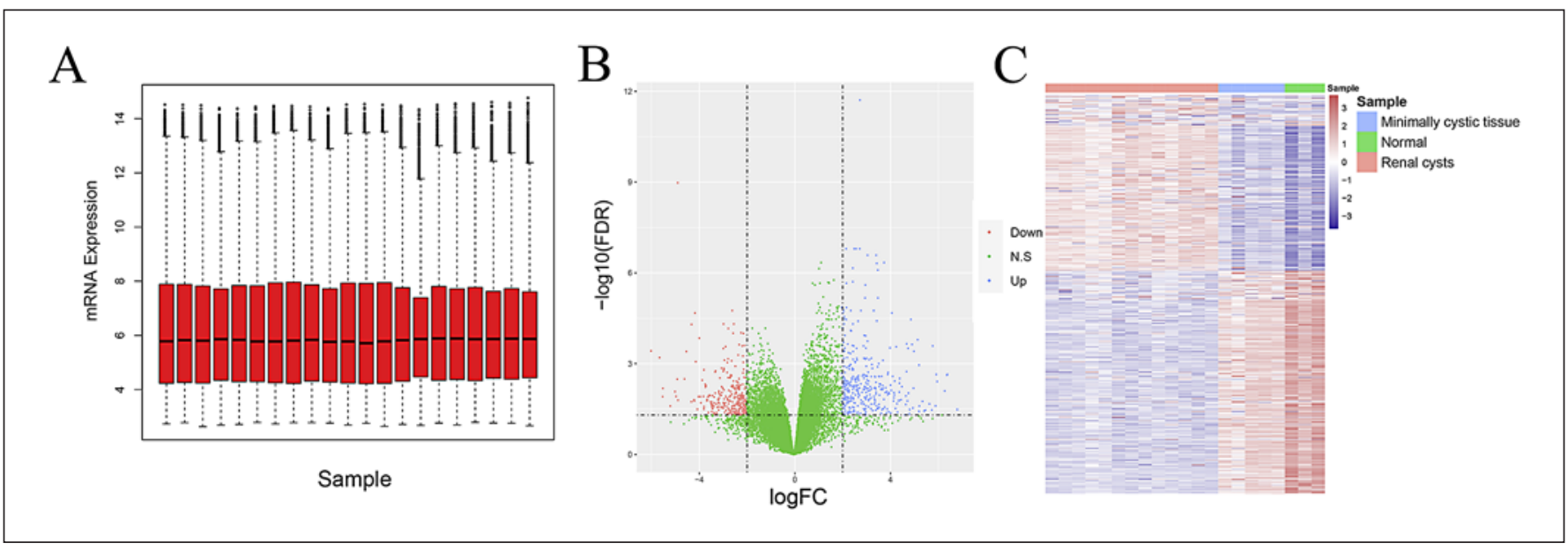

Fig. 1. The analysis of differential gene for PKD. A The standardization of the gene chip data. B The volcano map of differentially expressed genes. The horizontal axis represents the multiple of differential expression ( $\log 2 \mathrm{FC}$ ), the vertical axis represents $-\log 10$ (FDR), while the blue dot represents 363 upregulated genes, and the red dot represents 286 downregulated genes, respectively. C The heat map of differentially expressed genes. The horizontal axis indicates the sample, vertical axis indicates different genes, while red is high gene expression, and blue is low gene expression, respectively. $\mathrm{PKD}$, polycystic kidney disease.
TATAGGTTGCTGGCGGTTCTCTTCGTTTTAGAGCTAGAAATAGC-3' (upstream) and 5' -AAAAGCACCGACTCGGTGCCACTTTTTCAAGTTGATAACGGACTAGCCTTATTTTAACTTGCTATTTCTAGCTCTAAAAC-3' (downstream), respectively. A total of $400 \mathrm{pg}$ Cas 9 mRNA and $100 \mathrm{pg}$ pi4kb gRNA were co-injected into zebrafish embryos at 1-cell stage. This approach finally yielded 2 PI $4 K B$ loss-of-function alleles (pi $4 \mathrm{~kb} \Delta 1$ with 1-bp deletion and pi4kb $\Delta 5$ with 5 -bp deletion in exon 2 ), resulting in predicted truncation of PI4KB at amino acid position 330 th (pi4kb $\Delta 1$ ) or 334th (pi4kb $\Delta 5$ ) in the PI4KB depletion mutants. Paraffin sections of PI4KB depletion mutants and sibling controls were compared for PKD phenotype and diameter of inner tubules, respectively.

\section{Cell Culture and Plasmid Transfection}

Human normal renal epithelial cell line HK-2 was purchased from the Cell Bank of the Chinese Academy of Sciences (Beijing, China). The cells were transfected with negative control vector, PI4KB siRNAs [20], and GNAS siRNAs as we previously reported [21].

\section{Western Blotting Analysis}

HK-2 cells were harvested and washed twice with PBS. The cell lysis buffer contained 25 mM HEPES pH 7.5, $150 \mathrm{mM} \mathrm{NaCl}, 0.5 \%$ NP-40, and $0.1 \%$ SDS plus proteinase inhibitor cocktail (SigmaAldrich, St. Louis, MO, USA). The total protein concentration of cell lysate was determined using the Pierce BCA protein assay kit (Thermo Fisher Scientific, Beijing, China). Then, $30 \mathrm{mg}$ of proteins were loaded on premade 8-15\% SDS polyacrylamide gel (Invitrogen) for separation and then electrotransferred onto a nitrocellulose membrane. The blots were incubated in PBS buffer with $5 \%$ defat milk and $0.02 \%$ Tween 20 at room temperature for $1 \mathrm{~h}$ before primary antibody incubation overnight at $4^{\circ} \mathrm{C}$. Horseradish peroxidase-conjugated secondary antibody (Jackson Immunore- search Laboratory) incubation was conducted at room temperature for $1 \mathrm{~h}$. SuperSignal West Pico Chemiluminescent Substrate (Pierce; Thermo Scientific) was used for visualization of immunoreactive proteins.

\section{Statistical Analysis}

The Student $t$ test was used to compare the differences between PI4KB mutants and sibling controls, with $p<0.05$ as the significant threshold. The statistical analysis was established by R software, with version number v3.5.2.

\section{Results}

\section{Differentially Expressed Gene Analysis Results}

We first standardized the data of the gene chip to obtain biologically significant changes in gene expression. We found that the data after standardization had less deviation (Fig. 1A). Using the normalized data to analyze the differential genes, a total of 649 differentially expressed genes were demonstrated in the control group relative to the polycystic kidney patient samples, including 363 upregulated genes and 286 downregulated genes (Fig. 1B), At the same time, the expression levels of the selected genes were significantly different between the 2 groups (Fig. 1C). Among these genes, PIAKB, GNAS, UMOD, SLC7A13, and MIOX were more prominent, which indicated they were crucial for PKD development. 


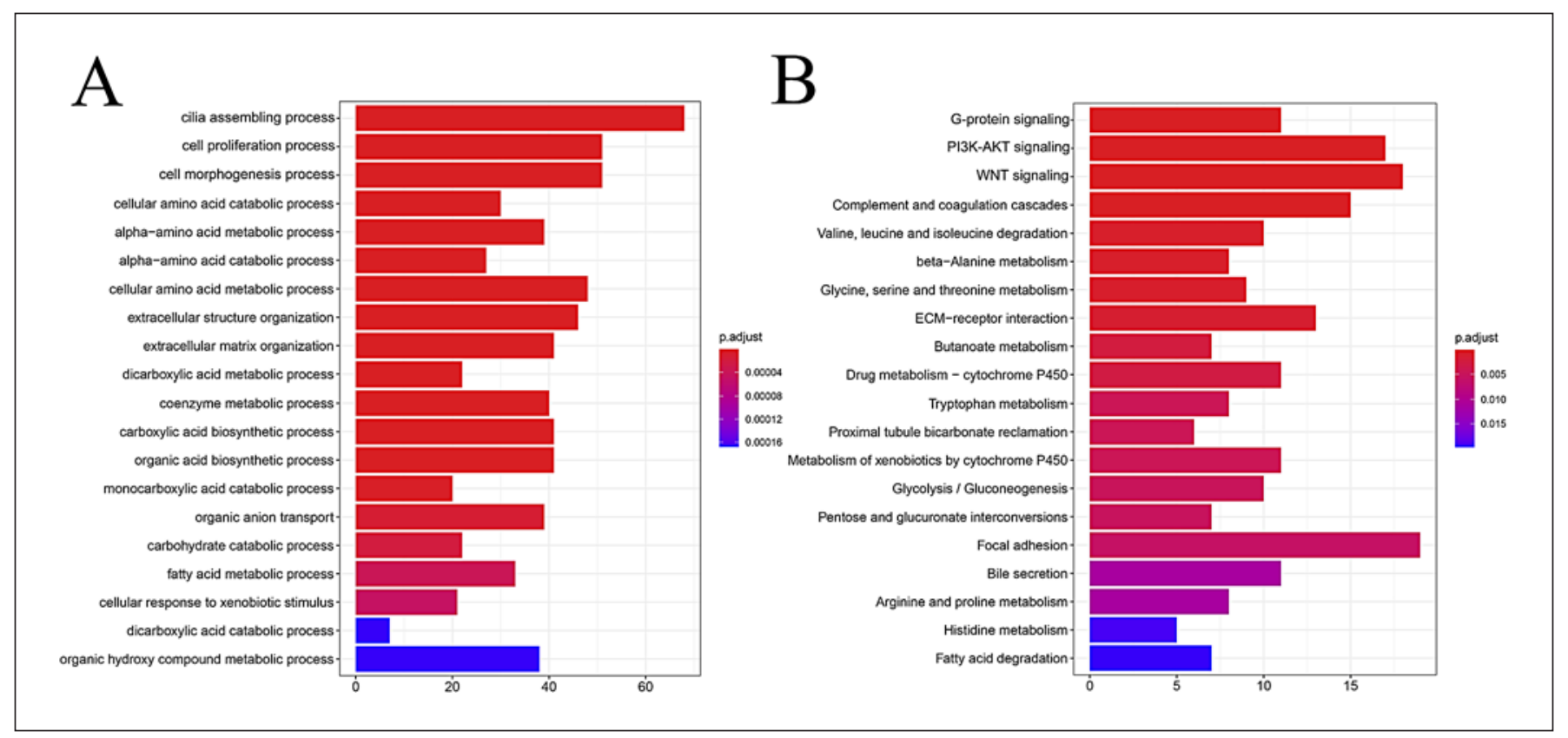

Fig. 2. GO and KEGG enrichment results for PKD. A The top 20 GO term enrichment results with the largest number of genes. In the figure, the horizontal axis represents the number of enriched genes, and the vertical axis represents the name of each GO term, respectively. B The enrichment results of the 20 KEGG pathways

\section{GO and KEGG Enrichment Analysis Results}

By performing GO and KEGG enrichment analysis on these 649 differentially expressed genes, we found that these differentially expressed genes were enriched in GO terms related to various biological processes such as cilia assembling, cell proliferation, and cell morphogenesis (Fig. 2A). At the same time, the G-protein signaling, PI3K-AKT signaling, and WNT signaling pathways were significantly enriched in KEGG pathway analysis, suggesting those pathways were closely associated with PKD formation (Fig. 2B).

\section{PPI Network Construction and Screening of Hub \\ Genes}

We established a PPI network of 649 genes by the STRING database as 321 genes with a confidence score $\geq 0.4$. We used Cytoscape software to analyze the topological structure of the PPI network and utilized the MNC algorithm to score the importance of each node in the network for the primary 50 genes selection. As a primary key regulator selected from $P K D$ patients, the $P I 4 K B$ gene demonstrated the potential connections with GNAS and AKT (Fig. 3). with the largest number of genes. The horizontal axis in the figure indicates the number of genes enriched, and the vertical axis indicates the name of each KEGG pathway, respectively. PKD, polycystic kidney disease.

\section{Verification of PI4KB Function in Ciliogenesis for $P K D$ Formation}

From the result above, we have shown that $P I 4 K B$ and GNAS were potential hub genes for kidney cyst development. Next, we sought to explore the function of PI4KB using a zebrafish $P I 4 K B$ loss-of-function mutant model. As shown in Figure 4A, the depletion of PI4KB caused a PKD phenotype in $P I 4 K B$ mutants (shown as dilated tubules in Fig. 4A). The inner tubule diameters of $P I 4 K B$ mutants $(n=20,74.21 \pm 23.66 \mu \mathrm{m})$ were significantly different compared with sibling controls $(n=20,24.24 \pm$ $20.68 \mu \mathrm{m})$ (Fig. 4B). More importantly, primary cilia especially the single cilia were totally absent in PI4KB mutants (Fig. 4C). Previously, our results suggested that GNAS was critical for pronephric cyst formation [22]. Since PPI network data also implied the interaction between GNAS and PI4KB, we further investigated the relation between them by Western blotting analysis. It could be seen that the protein level of PI4KB was repressed in GNAS knockdown HK-2 cells (Fig. 4D). Meanwhile, although the protein level of $\mathrm{t}$-Akt was unaffected, p-Akt was dramatically decreased in the PI4KB loss-of-function cell line (Fig. 4E). This was consistent with GNAS depletion (Fig. 4F). 


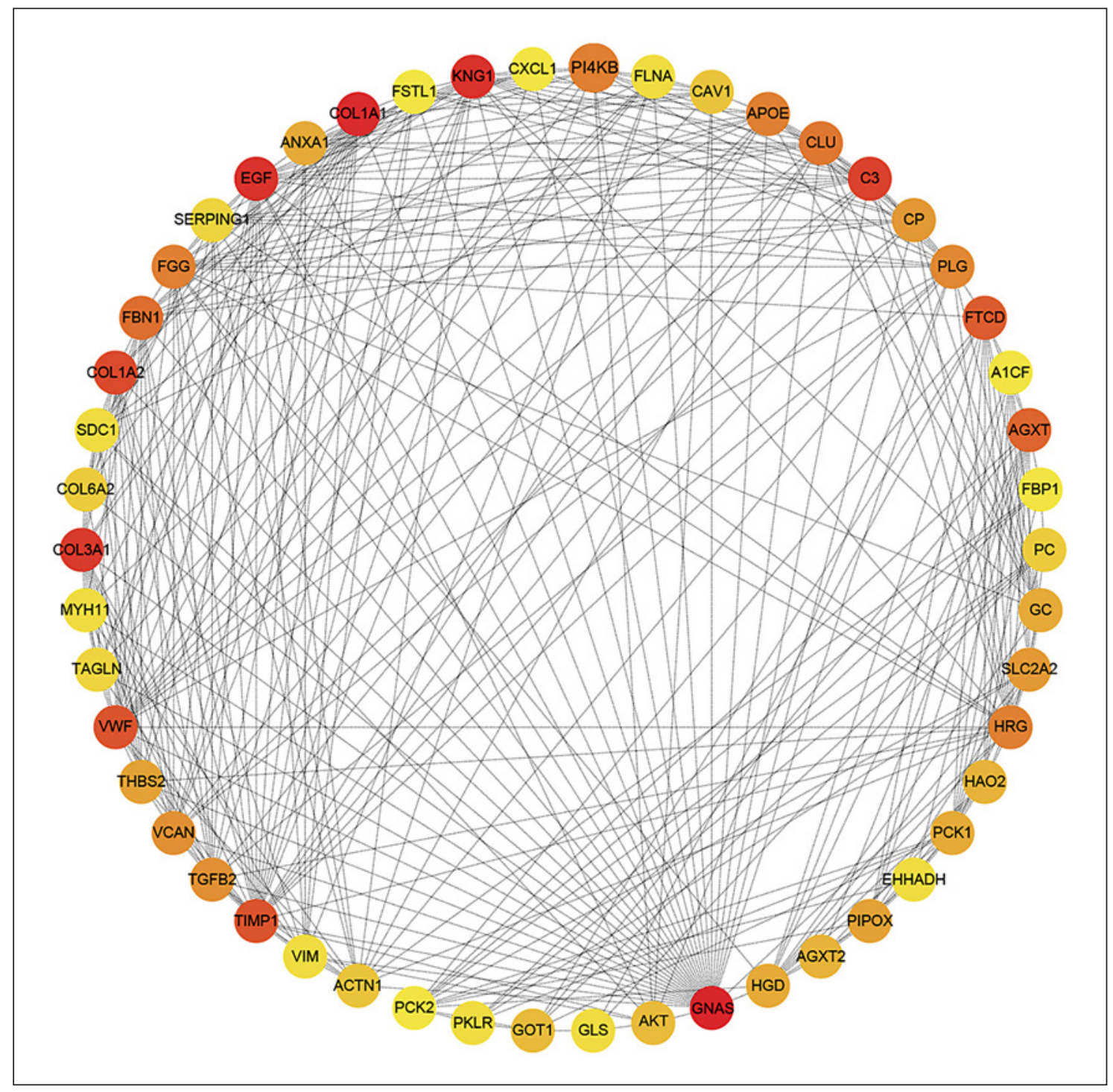

Fig. 3. The PPI network diagram of key genes related to PKD. Each dot represents a node. PPI, protein-protein interaction; PKD, polycystic kidney disease.

\section{Discussion}

PKD is one of the most common diseases for humans, which affects not only the kidney but also other organs including the liver [23]. Until now, researchers have been trying to find breakthroughs in the prevention, early screening, diagnosis, and treatment of PKD. Even with some progress in these areas, the efficiency is still inferior, which is mainly due to the complexity and diversity of molecular mechanisms behind the disease [24]. PC1 has always been considered as a noncanonical G-protein sig- naling receptor (GPCR), which is based on the structural as well as functional analysis of the large protein [25]. To this end, G-protein-related signaling has become one interesting feature for the PKD study. For instance, the vasopressin V2 receptors (VPV2R, a GPCR) have been shown to manipulate cAMP levels in the distal nephron and collecting duct, a major site of ADPKD cysts. The VPV2R antagonists have been testified to inhibit cyst development in both animal model and human clinical trial. Indeed, the tolvaptan, a VPV2R inhibitor, has passed the phase II clinical trials and initiated a phase III double- 


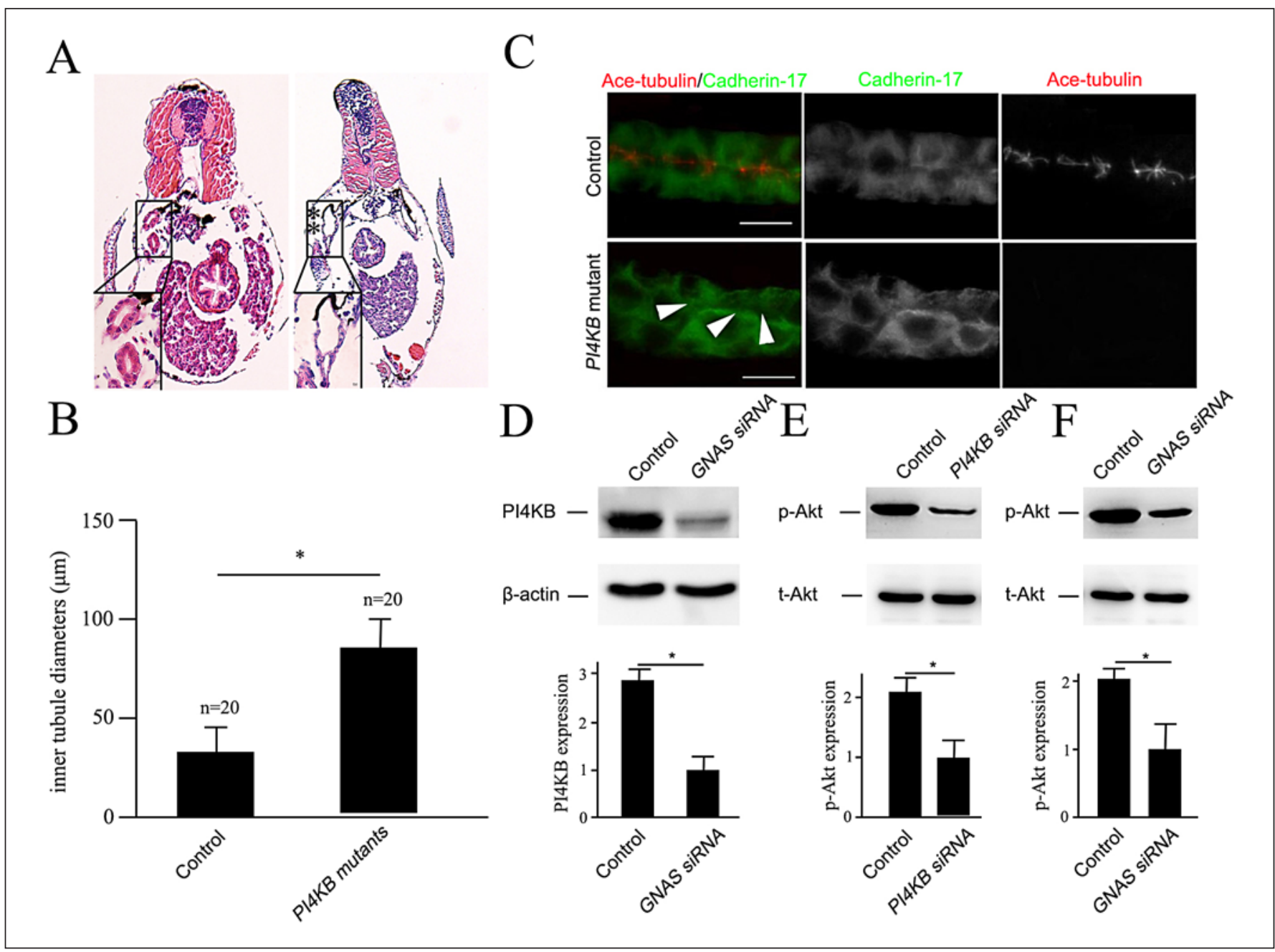

Fig. 4. Functional verification of PI4KB for PKD. A The PKD phenotypes generated by $P I 4 K B$ mutants. The asterisk indicates dilated tubules. B The statistical analysis for PKD phenotypes compared mutants with sibling controls. $C$ The immunostaining of primary cilia for $P I 4 K B$ mutant zebrafish. The white arrow represents the absence of single cilia in the pronephric duct of mutant zebrafish. D Western blotting and corresponding densitometry analysis demonstrate the expression level of PI4KB in the control vector and GNAS siRNA-transfected HK-2 cells. E, F Western blotting shows the activity change of $\mathrm{t}-\mathrm{Akt}$ and $\mathrm{p}$-Akt in the control vector, PI4KB siRNA, and GNAS siRNA-transfected HK-2 cells, respectively. The asterisk indicates $p<0.05$. t-Akt, total Akt; p-Akt, phospho Akt. blind placebo-controlled trial, which is one of the few potential chemotherapy for PKD treatment [26].

Previously, using a pronephric kidney of Xenopus as a model system, we supported the close association between G-protein and PC1 based on the evidence that the G-protein-binding domain is crucial for PC1 function, and PC1 has a high binding affinity as a classical GPCR with several G-proteins, especially GNAS [22]. Subsequently, we observed the significantly increased expression of GNAS in the renal cell carcinoma, which confirmed the function of GNAS for renal cell proliferation through a classical G-protein signaling with the involvement of PKA [21]. Since aberrant cell proliferation is also an indication for PKD, these results demonstrated the key functions of GNAS for kidney cyst formation. Here, in this study, we established a differentially expressed genes profile for PKD patients compared with control specimens. Within these genes, GNAS and PI4KB were the prominent ones. With further investigations, PI4KB might function as a downstream factor. Previously, Dr. Zhang and his colleagues [21] found that the lack of PI4KB caused loss of hair cells in the otic vesicles as well 
Fig. 5. Schematic illustration of the signaling model from this study.

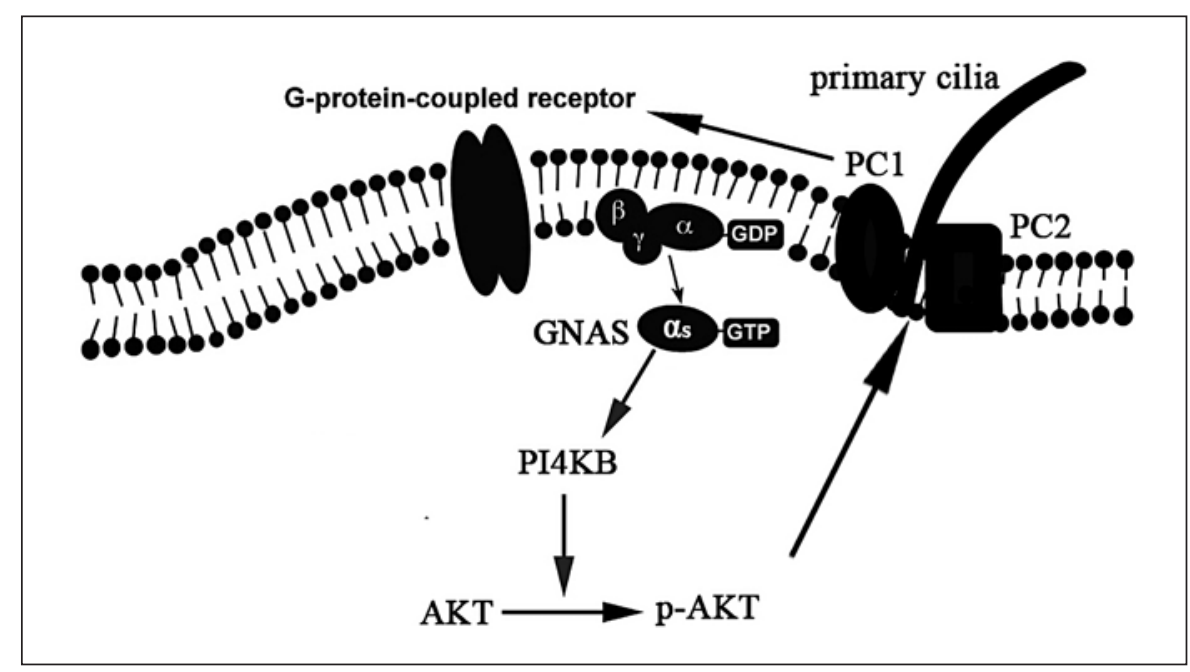

as in the lateral line neuromasts of zebrafish [20]. This was in line with the clinical findings that human PI4KB mutations caused hearing disability and congenital deafness. It is well known that PI4KB is mainly responsible for the synthesis of PI4P, which in turn contributes to synthesize PI $(4,5)$ P2 and PI $(3,4,5)$ P3. Previously, Xu and his colleagues [15] have proved that the apical PI $(4,5) \mathrm{P} 2$ was required for ciliogenesis and suppression of polycystic kidney disease. In this study, we extended the signaling model to upstream factors (PI4KB and GNAS), which generated similar phenotypes. All of these outcomes hypothesized a cilia-associated innovative signaling axis with PI4KB as a central position. The PKD patient source we analyzed in this study was initiated by Song et al. [27]. In that work, they suggested that the Wnt signaling pathway and renal cysts represent metabolic pathways (e.g., amino acid, fatty acid, urea cycle, and ATP metabolism) and were significantly enriched in PKD patients, which was similar to the results shown here (Fig. 2). At the same time, some hub genes including UMOD, SLC7A13, and MIOX were suggested by both studies. Moreover, they claimed that the genetic network behind the disease was extremely complicated, of which a variety of signaling pathways were closely involved (Fig. 3 in [27]). However, they evaluated differentially expressed genes based on a mixture of cell types, and they were not able to assess the level or activity of specific protein. Based on our previous publications, we focused on the G-protein and cilia-associated genes for the following analysis. Meanwhile, we utilized (CRISPR/Cas9) system genome-editing strategy to target single specific hub gene, and the corresponding protein activity was also measured here. Nevertheless, some other potential candidates (like the factors in ERK,
mTOR, and PI3K signaling cascades) are worthy of further in-depth investigation.

Primary cilia have a filiform microtubular structure, anchored in the basal body and extending from the apical membrane into the tubular lumen. Primary cilia have been suggested to play an important role in embryonic development as well as in postnatal life, dysfunction of which is associated with situs inversus, retinal abnormalities, impaired mucociliary clearance, infertility, hydrocephalus, and PKD [28]. In the renal epithelial cells, primary cilia have been shown to function as a flow sensor and respond to flow-mediated bending with a prolonged intracellular calcium increase. In the analysis here, we also confirmed the interaction between cilium assembling pathway with PKD development. With the functional experiments, we explored the molecular mechanism for the connection, which initiated a primary signaling model: PC1 is a classical GPCR, associated with GNAS (a key G-protein), which inversely manipulates the activity of PI4KB and subsequently functions by recruiting and phosphorylating Akt into p-Akt for the ciliogenesis in PKD (see Fig. 5 for model detail).

In addition to the potential model establishment for $\mathrm{PKD}$, another feature of this study was to explore multiple key associated genes. Genome-wide association studies have uncovered that UMOD is among the most outstanding loci associated with chronic kidney disease (CKD) in the general population, because it has a large effect on eGFR and CKD risk that is consistent across different ethnic groups [29]. The membrane protein AGT1/SLC7A13 is the second partner of rBAT, which is a heterodimeric amino acid transporter responsible for epithelial transport [30]. MIOX stands for the proximal tubular enzyme myo-inosi- 
tol oxygenase. It has been suggested that MIOX overexpression exacerbates, whereas MIOX gene disruption protects against cisplatin-induced AKI [31]. Besides these, the connections between these genes and PKD are not fully understood yet. All of these deserve further investigation.

\section{Conclusion}

Collectively, in the light of the fact that there remains no gold standard treatment for PKD, we established an innovative differentially expressed gene profile for $\mathrm{PKD}$. For the potential gene candidates (GNAS and PI4KB), we further explored the intrinsic connection with ciliogenesis as well as PKD formation. Using an animal mutant model, the functions of PI4KB in PKD were better understood, and a comprehensive signaling model was developed in-depth. Overall, we shed light on questions and challenges posed by the $\mathrm{PKD}$, which provided a beneficial reference for future understanding of the disease.

\section{Statement of Ethics}

There is no human sample used in this study except for chips in the bioinformatics analysis. So, ethical approval is not applicable.

\section{Conflict of Interest Statement}

The authors declare no competing interests.

\section{Funding Sources}

This research was funded by the National Natural Science Foundation of China (Grant No. 82070687 to Bo Zhang) and the Natural Science Foundation of Tianjin (Grant No. 16JCQNJC12100 to Bing Yang, 18JCTPJC60300 and 18JCYBJC93000 to Xuchun Che, and 19JCYBJC26300 to Lei Zhi) .

\section{Author Contributions}

Ling Lu, Qiuling Liu, and Jingjing Zhang analyzed the data. Lei Zhi and Xuchun Che performed the cell experiments. Bo Xiao and Mingxuan Cui developed the enrichment data and PPI results. Mingyu Yu and Bing Yang performed the Western blotting. Bo Zhang supervised the project and wrote the manuscript.

\section{Data Availability Statement}

The datasets used or analysed during the current study are available from the corresponding author on reasonable request.

\section{References}

1 Bergmann C, Guay-Woodford LM, Harris PC, Horie S, Peters DJM, Torres VE. Polycystic kidney disease. Nat Rev Dis Primers. 2018; 4(1):50.

2 Bergmann C. Recent advances in the molecular diagnosis of polycystic kidney disease. Expert Rev Mol Diagn. 2017;17(12):1037-54.

3 Ghata J, Cowley BD Jr. Polycystic kidney disease. Compr Physiol. 2017;7(3):945-75.

4 Kim DY, Park JH. Genetic Mechanisms of ADPKD. Adv Exp Med Biol. 2016;933:13-22. Kotsis F, Boehlke C, Kuehn EW. The ciliary flow sensor and polycystic kidney disease. Nephrol Dial Transplant. 2013;28(3):518-26.

5 Ma S, Guan KL. Polycystic kidney disease: a hippo connection. Genes Dev. 2018;32(1112):737-9.

6 Torres VE, Harris PC, Pirson Y. Autosomal dominant polycystic kidney disease. Lancet. 2007;369(9569):1287-301.

7 Wilson PD. Polycystic kidney disease. N Engl J Med. 2004;350(2):151-64.

8 Cornec-Le Gall E, Alam A, Perrone RD. Autosomal dominant polycystic kidney disease. Lancet. 2019;393(10174):919-35.
9 Chebib FT, Torres VE. Recent advances in the management of autosomal dominant polycystic kidney disease. Clin J Am Soc Nephrol. 2018;13(11):1765-76.

10 Bangs F, Anderson KV. Primary cilia and mammalian hedgehog signaling. Cold Spring Harb Perspect Biol. 2017;9(5):a028175.

11 Satir P. CILIA: before and after. Cilia. 2017;6: 1.

12 Anvarian Z, Mykytyn K, Mukhopadhyay S, Pedersen LB, Christensen ST. Cellular signalling by primary cilia in development, organ function and disease. Nat Rev Nephrol. 2019; 15(4):199-219.

13 Hua K, Ferland RJ. Primary cilia proteins: ciliary and extraciliary sites and functions. Cell Mol Life Sci. 2018;75(9):1521-40.

14 Chalupska D, Eisenreichova A, Rozycki B, Rezabkova L, Humpolickova J, Klima M, et al. Structural analysis of phosphatidylinositol 4-kinase IIIbeta (PI4KB) 14-3-3 protein complex reveals internal flexibility and explains 14-3-3 mediated protection from degradation in vitro. J Struct Biol. 2017;200(1):36-44.

$15 \mathrm{Xu} \mathrm{W}$, Jin M, Huang W, Wang H, Hu R, Li J, et al. Apical PtdIns $(4,5)$ P2 is required for ciliogenesis and suppression of polycystic kidney disease. FASEB J. 2019;33(2):2848-57.
16 Ritchie ME, Phipson B, Wu D, Hu Y, Law CW, Shi W, et al. Limma powers differential expression analyses for RNA-sequencing and microarray studies. Nucleic Acids Res. 2015; 43(7):e47.

17 Yu G, Wang LG, Han Y, He QY. Clusterprofiler: an R package for comparing biological themes among gene clusters. OMICS. 2012; 16(5):284-7.

18 Szklarczyk D, Gable AL, Lyon D, Junge A, Wyder S, Huerta-Cepas J, et al. STRING v11: protein-protein association networks with increased coverage, supporting functional discovery in genome-wide experimental datasets. Nucleic Acids Res. 2019;47(D1):D60713.

19 Shannon P, Markiel A, Ozier O, Baliga NS, Wang JT, Ramage D, et al. Cytoscape: a software environment for integrated models of biomolecular interaction networks. Genome Res. 2003;13(11):2498-504.

20 Feng Y, Yu P, Li J, Cao Y, Zhang J. Phosphatidylinositol 4-kinase $\beta$ is required for the ciliogenesis of zebrafish otic vesicle. J Genet $\mathrm{Ge}$ nomics. 2020;47(10):627-36. 
21 Zhang B, Sun N, Mu X, Zhi L, Zhai L, Jiang Y, et al. $G$ protein alpha $S$ subunit promotes cell proliferation of renal cell carcinoma with involvement of protein kinase A signaling. DNA Cell Biol. 2017;36(3):237-42.

22 Zhang B, Tran U, Wessely O. Polycystin 1 loss of function is directly linked to an imbalance in G-protein signaling in the kidney. Development. 2018;145(6):dev158931.

23 Avasthi P, Maser RL, Tran PV. Primary cilia in cystic kidney disease. Results Probl Cell Differ. 2017;60:281-321.

24 Hama T, Park F. Heterotrimeric G protein signaling in polycystic kidney disease. Physiol Genomics. 2016;48(7):429-45.
25 Trudel M, Yao Q, Qian F. The role of G-protein-coupled receptor proteolysis site cleavage of polycystin-1 in renal physiology and polycystic kidney disease. Cells. 2016;5(1):3.

26 Harris PC, Torres VE. Polycystic kidney disease. Annu Rev Med. 2009;60:321-37.

27 Song X, Di Giovanni V, He N, Wang K, Ingram A, Rosenblum ND, et al. Systems biology of autosomal dominant polycystic kidney disease (ADPKD): computational identification of gene expression pathways and integrated regulatory networks. Hum Mol Genet. 2009;18(13):2328-43.

28 Kotsis F, Nitschke R, Boehlke C, Bashkurov M, Walz G, Kuehn EW. Ciliary calcium signaling is modulated by kidney injury molecule-1 (Kim1). Pflugers Arch. 2007;453(6): 819-29.
29 Devuyst O, Pattaro C. The UMOD locus: insights into the pathogenesis and prognosis of kidney disease. J Am Soc Nephrol. 2018;29(3): 713-26.

30 Olschok K, Vester U, Lahme S, Kurth I, Eggermann T. No evidence for point mutations in the novel renal cystine transporter AGT1/ SLC7A13 contributing to the etiology of cystinuria. BMC Nephrol. 2018;19(1):278.

31 Dutta RK, Kondeti VK, Sharma I, Chandel NS, Quaggin SE, Kanwar YS. Beneficial effects of myo-inositol oxygenase deficiency in cisplatin-induced AKI. J Am Soc Nephrol. 2017; 28(5):1421-36. 